\title{
Sphere-Function-Based Shape Modelling of Open Cell Metal Foam with Plateau Borders
}

\author{
Yuya Hanaoka ${ }^{1, *}$, Taku Itoh $^{2}$, Susumu Nakata ${ }^{3}$, Keiko Watanabe $^{4}$ \\ ${ }^{1}$ Graduate School of Industrial Technology, Nihon University, Japan \\ ${ }^{2}$ College of Industrial Technology, Nihon University, Japan \\ ${ }^{3}$ College of Information Science and Engineering, Ritsumeikan University, Japan \\ ${ }^{4}$ College of Science and Engineering, Ritsumeikan University, Japan \\ *ciyu19013@g.nihon-u.ac.jp
}

Received: January 7, 2020; Accepted: February 10, 2020; Published: April 23, 2020

\begin{abstract}
To construct an open cell metal foam model, a simple method using spheres has been proposed. In this method, spheres are arranged so that the edges are constructed as Plateau borders, and an implicit surface $f(\boldsymbol{x})=0$ combined by constructive solid geometry is generated from these spheres. The open cell metal foam model generated by the proposed method has edges and junctions, and the cross-sections of Plateau borders in the model are concave triangles. In the proposed method, the open cell model that has a periodic structure can be generated by adjusting the arrangement of spheres. In addition, by employing the $p$ norm-based implicit surfaces, the proposed method has been extended so that various kinds of open cell model can be constructed. Although the open cell models generated by the proposed method are midair models, they can be converted to solid models constructed as voxel meshes. Furthermore, an arbitrary shape defined by an implicit surface $g(\boldsymbol{x})=0 \mathrm{can}$ be represented as a collection of extracted cells in the voxel mesh of open cell model.
\end{abstract}

Keywords: Implicit surfaces, Open cell metal foams, Plateau borders, CSG

\section{Introduction}

Metal foams are mainly distinguished as two types by the state of cell structure, i.e., closed and open cells (see Figs. 1 and 2). In the closed cell, each cell is covered with a wall, i.e., each cell is isolated. On the other hand, the open cell is constructed by edges. Metal foams have characteristics such as light weight and high capability of shock-absorbing [1]. Since these characteristics are valuable, metal foams are expected to be applied to many fields of engineering, e.g., crash absorbers, and materials of trains and architecture [2]. For this reason, evaluation of the performance of metal foams by numerical simulation is required.

To apply metal foams to numerical simulations, modelling methods based on implicit surfaces [3] have been proposed [4, 5]. The method described in [4] has focused on the 


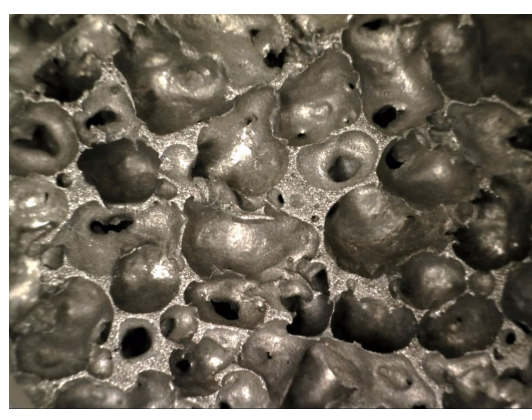

Figure 1: Closed cell metal foam.

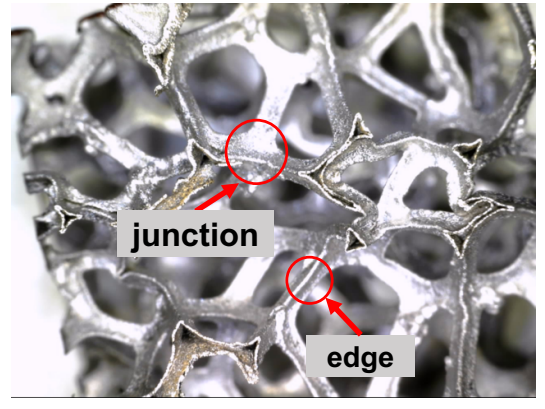

Figure 2: Open cell metal foam.

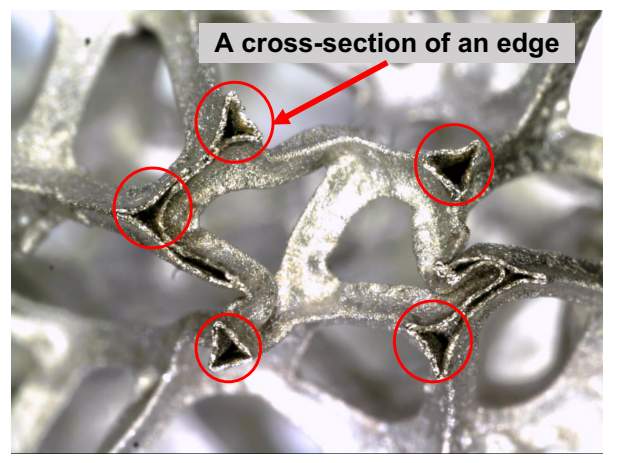

Figure 3: An enlarged view of an open cell structure.

modelling of the closed cells, and a method for generating an open cell model has not been touched. On the other hand, the method described in [5] can generate both models of closed and open cells. In this paper, we focus on open cell metal foams, and consider generating a method for modelling them by a different strategy from [5]. By limiting to open cell metal foams, there is a possibility that an algorithm based on sphere functions can be constructed. Note that the open cell metal foams have edges whose shape is distinctive. The edges are called Plateau borders [6]. The purpose of this study is to construct open cell metal foam models with Plateau borders. By employing the sphere-function-based algorithm, the Plateau borders can be represented.

The article is structured as follows. Section 2 introduces characteristics of open cell structure. Section 3 describes an open cell shape modelling using sphere functions. To flexibly construct an open cell model for numerical simulations, Section 4 describes procedures for generating an open cell model that has a periodic structure. Since the open cell model described in Sections 3 and 4 is midair one, a method for converting the midair model to solid one is shown in Section 5. Finally, some conclusions are shown in Section 6.

\section{Structure of Open Cell}

Closed cell metal foams are separated by thin walls. However, open cell metal foams do not contain these walls. The structure of a real open cell metal foam is shown in Fig. 2. 


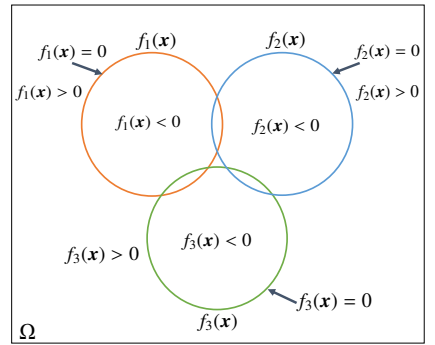

(a)

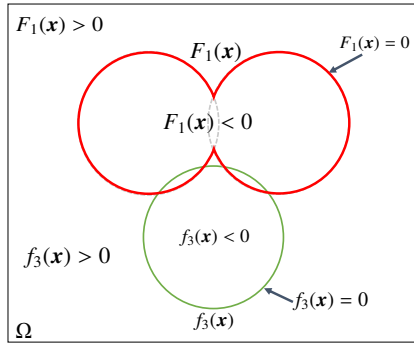

(b)

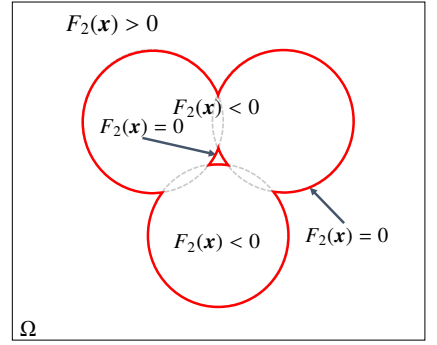

(c)

Figure 4: Schematic view of (a) initial arrangement of sphere functions $f_{1}(\boldsymbol{x}), f_{2}(\boldsymbol{x})$ and $f_{3}(\boldsymbol{x})$, (b) $F_{1}(\boldsymbol{x})=\min \left\{f_{1}(\boldsymbol{x}), f_{2}(\boldsymbol{x})\right\}$ and $f_{3}(\boldsymbol{x})$, and (c) $F_{2}(\boldsymbol{x})=\min \left\{F_{1}(\boldsymbol{x}), f_{3}(\boldsymbol{x})\right\}$ in the strategy for constructing a Plateau border.

As shown in this figure, open cell metal foams are constructed by edges and junctions. The edge is generally called Plateau border [6], and Plateau borders are connected in a junction. Widths of junctions tend to be wider than those of edges. The cross-sections of the edges are shown in red circles of Fig. 3. Note that the cross-sectional shape of Plateau borders is a concave triangle (see Fig. 3). Although the open cell metal foams shown in Figs. 2 and 3 are midair types, solid ones also exist such as shown in [7].

In this study, to represent a shape of Plateau border, we arrange spheres appropriately. In addition, the shape is constructed by combining the spheres using the constructive solid geometry (CSG) [8].

\section{Modelling of Open Cell Metal Foam}

\subsection{Implicit Function}

The function $f(\boldsymbol{x})$ is generated so that $f(\boldsymbol{x})$ has the following properties:

$$
\left\{\begin{array}{l}
f(\boldsymbol{x})<0, \quad \text { (inside of the surface), } \\
f(\boldsymbol{x})>0, \quad \text { (outside of the surface). }
\end{array}\right.
$$

Additionally, in this study, to generate a open cell model, we employ a sphere, thus an implicit function $f(\boldsymbol{x})$ is represented as follows:

$$
f(x)=(x-a)^{2}+(y-b)^{2}+(z-c)^{2}-r^{2}
$$

where $(a, b, c)$ is the center point of sphere and $r$ its radius.

\subsection{Combining Spheres by CSG}

In this study, to construct shapes of Plateau borders, we describe a strategy for combining spheres using CSG. Figure 4(a) shows the schematic view of spheres arrangement. These 


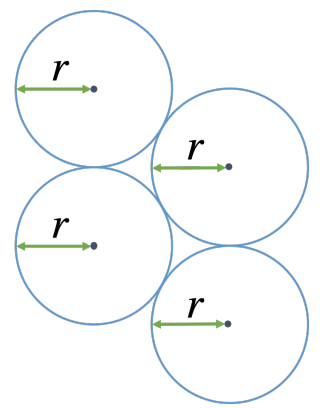

(a)

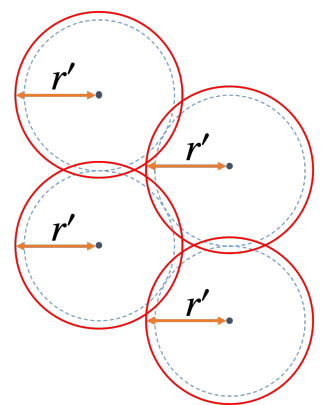

(b)

Figure 5: Schematic view of sphere arrangements for (a) Step 1 and (b) Step 2 described in Section 3.2. Here, $r^{\prime}=r+\alpha$.

spheres are represented scalar fields by $f_{1}(\boldsymbol{x}), f_{2}(\boldsymbol{x})$ and $f_{3}(\boldsymbol{x})$ respectively. Note that each line in Fig. 4(a) is the implicit surface from each scalar field.

$$
F_{1}(\boldsymbol{x})=\min \left\{f_{1}(\boldsymbol{x}), f_{2}(\boldsymbol{x})\right\}
$$

Figure 4(b) shows the scalar field $F_{1}(x)$ generated by (3). Note that $F_{1}(x)=0$ is represented by red line in Fig. 4(b). Similarly, a scalar field $F_{2}(\boldsymbol{x})$ is generated from $F_{1}(\boldsymbol{x})$ and $f_{3}(\boldsymbol{x})$. As a result, we obtain the scalar field $F_{2}(x)$ shown in Fig. 4(c). Note that the red line in the same figure is represented the implicit surface, $F_{2}(\boldsymbol{x})=0$. In the surface, we consider the shape surrounded by spheres becomes Plateau borders. This is because the shape is a concave triangle [6].

Next, we describe a technique for arrangement of spheres to construct an open cell model. To obtain a surface whose edges have a Plateau border shape, the spheres have to be arranged so that parts of their surface have intersection. By the following 2 steps, an appropriate arrangement of spheres can be obtained.

(Step 1) Spheres of radii $r$ are arranged so that adjacent spheres touch each other as shown in Fig. 5(a).

(Step 2) All sphere centers are maintained and new radii $r^{\prime}$ are set by $r^{\prime}=r+\alpha$, as shown in Fig. 5(b).

Here, $\alpha$ is a parameter for determining the amount of intersection of spheres. A construction method of an open cell model using the strategy is described in the next section.

\subsection{Constructing Open Cell Model}

In the strategy of Section 3.2, arranged spheres are combined by CSG. We focus on a shape surrounded by spheres (See the concave triangle in center of Fig. 4(c)). Note that, to generate an open cell model, spheres are arranged as shown in Fig. 6(a). Figure 6(b) shows an example of three-dimensional arrangement of spheres based on Fig. 6(a). By the strategy of section 3.2, we generate a combined surface of the arranged spheres and the combined result 


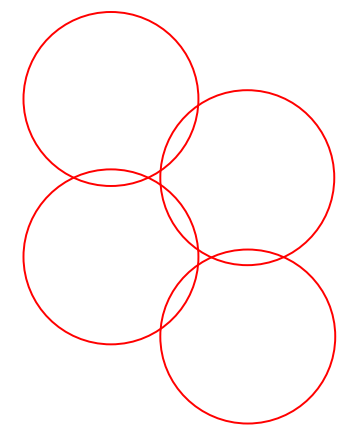

(a)

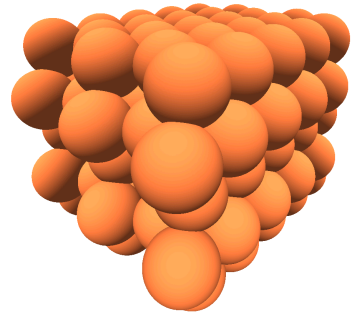

(b)

Figure 6: Examples of sphere arrangements for generating open cell structure in (a) 2-D and (b) 3-D cases.

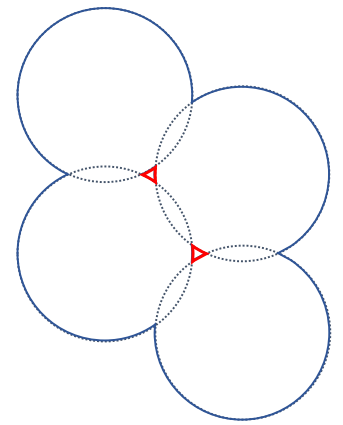

(a)

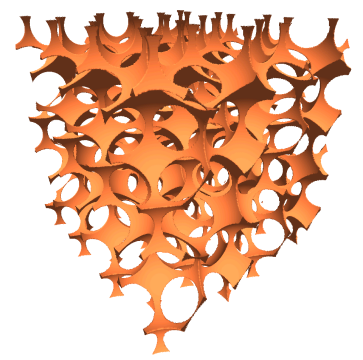

(b)

Figure 7: Results of (a) combining surfaces using the strategy of Section 3.2 and (b) an open cell model by the method of Section 3.3.

is shown in Fig. 7(a). In this figure, red and blue surfaces are found. In the proposed method, we employ only red surfaces for generating an open cell model, i.e. the blue surfaces are removed. A result of extracted surface is shown in Fig. 7(b).

We consider that the shape shown in this figure is a model of open cell metal foam generated by proposed method. Figure 8 shows Plateau borders and these junctions contained in the open cell model. We see from this figure that the shapes of Plateau borders in the model are similar to real one. Note that, for constructing models shown in this section, we set $r=2.0$ and $\alpha=0.15$ so that the Plateau borders can be constructed.

\subsection{Extension of the Proposed Method by $p$-norm}

In the previous section, we showed the proposed method based on sphere functions. The sphere functions can be generalized by $p$-norm, i.e., (2) is rewritten as

$$
f(\boldsymbol{x})=|x-a|^{p}+|y-b|^{p}+|z-c|^{p}-r^{p}
$$

where $p$ is a parameter for determining a shape of $p$-norm-based implicit surface, $f(\boldsymbol{x})=0$. Figure 9 shows the shapes depending on the value of $p$. By employing various values of $p$, 


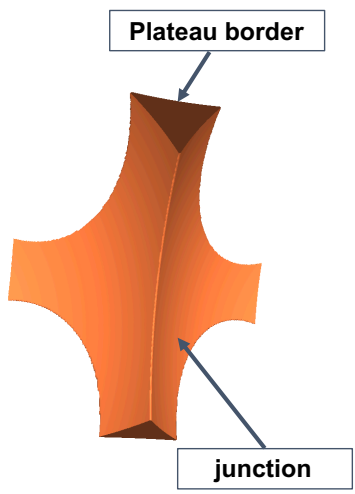

Figure 8: Shape of Plateau borders and a junction in the open cell model.

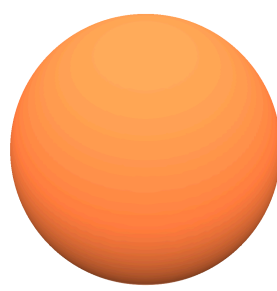

(a)

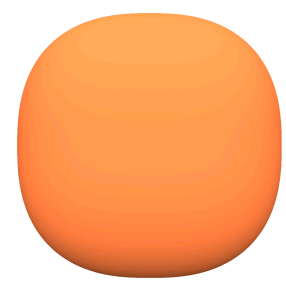

(b)

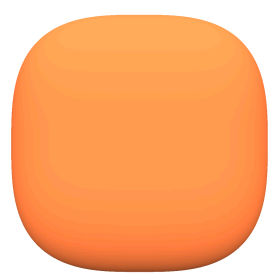

(c)

Figure 9: $p$-norm-based implicit surfaces, $f(\boldsymbol{x})=0$, for (a) $p=2.0$, (b) $p=2.5$ and (c) $p=3.0$.

the proposed method can be extended, i.e., the size of edges and junctions in a modelling result can be controlled by $p$. Modelling results for $p=2.0,2.1$ and 2.2 are shown in Table 1. In this table, enlarged views of the results corresponding to each of values $p$ are shown in the left side. In addition, in the right side of this table, the enlarged parts of edges and a junction extracted from the left figures. We see from this table that modelling results are deformed, depending on the value of $p$. Note that the shape of cross-section of edges retains the concave triangle, even $p$ is changed, if $p$ is in an appropriate range that can be constructed Plateau borders. For example, the range is $2.0 \lesssim p \lesssim 2.6$ for the case where $r=2.0$ and $\alpha=0.15$. Note that the range depends on the parameters $r$ and $\alpha$.

\section{Constructing Periodic Structure Model}

To flexibly construct an open cell model for numerical simulations, it is required that the model can be generated as an arbitrary shape. To this end, we first describe procedures for generating an open cell model that has a periodic structure in this section. Generating an arbitrary shape is described in Section 5.

The periodic structure is constructed based on the spheres arranged by the technique of Section 3.4. Figure 10 shows the arrangement of spheres for generating periodic structure. 
Table 1: Dependence of the generated open cell models on $p$. Left side is enlarged views of the models corresponding to each of values $p$, and right side is the enlarged parts of edges and a junction extracted from the left figures.

\begin{tabular}{|l|l|l|}
\hline$p$ & Enlarged view & Edges and junction \\
\hline 2.0 & & \\
\hline
\end{tabular}

The details of arrangement of this figure are shown in Fig. 11. Here, let the positive direction of $z$-axis be upward. The lower, middle, and upper parts of Fig. 10 correspond to Figs. 11(a), (b), and (c), respectively. In Fig. 11, the red dot denotes the basis points for the arrangement of spheres. The $x$ - and $y$-coordinate of basis points are the same in Figs. 11(a), (b), and (c), i.e., $x=x_{a}, y=y_{a}$. In addition, the spheres are arranged so that $z_{b}=z_{a}+2 \sqrt{6} r / 3$ and $z_{c}=z_{a}+4 \sqrt{6} r / 3$, where $z_{a}, z_{b}$ and $z_{c}$ denote $z$-coordinate of basis point in Figs. 11(a), (b), and (c), respectively. Note that $z_{a}$ can be set arbitrarily, and $z_{a}, z_{b}$ and $z_{c}$ are also $z$-coordinate of sphere centers in Figs. 11(a), (b), and (c), respectively.

Based on the arrangement shown in Fig. 10, an open cell model that has a periodic structure is generated as shown in Fig. 12. The model shown in this figure can be obtained by extracting the rectangular domain colored in Fig. 13. Note that Figs. 13(a), (b) and (c) 


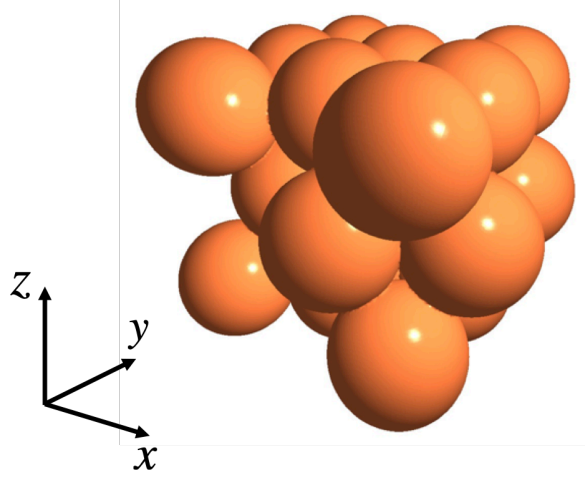

Figure 10: An arrangement of spheres for generating a unit model of periodic structure.

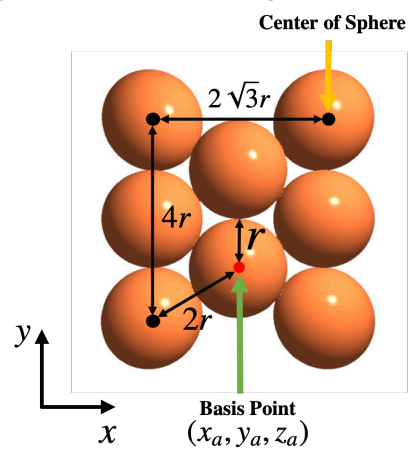

(a)

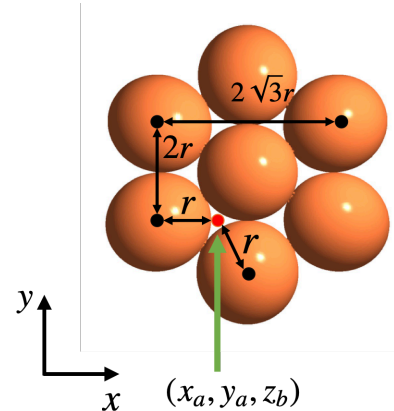

(b)

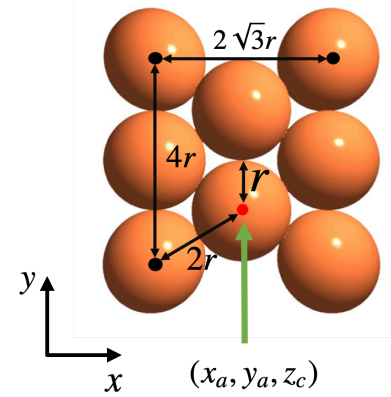

(c)

Figure 11: The arrangements of Fig. 10 for (a) lower, (b) middle and (b) upper parts.

are front, side and plan views of Fig. 10, respectively. The model shown in Fig. 12 becomes a unit model of periodic structure. Hence, the model can be connected as shown in Fig. 14. By connecting the unit model, we can obtain an open cell model that is arbitrarily extended as shown in Fig. 15.

Even for the case where $p$-norm-based implicit functions are employed for generating an open cell model, a unit model for a periodic structure can be constructed. If $p$ is a constant, then the unit model can be generated by the similar procedures for the case where spheres are employed. In addition, even if various values of $p$ are employed, a unit model can be generated by almost the same procedures. However, since it is required that the boundaries of $x$-, $y$ - and $z$-direction can be connected respectively, the same values of $p$ must be set in the connecting parts for each direction. Note that, as long as each of values $p$ is the same at the connecting parts, many kinds of $p$-norm-based implicit surfaces can freely be arranged and various unit models can be generated. 


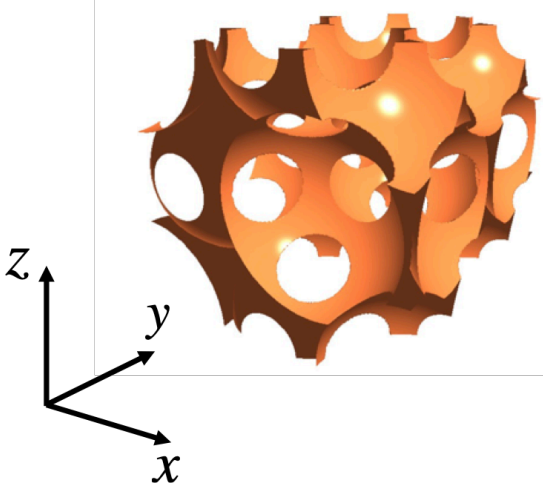

Figure 12: The unit model of open cell model that has a periodic structure based on the arrangement of Fig. 10.

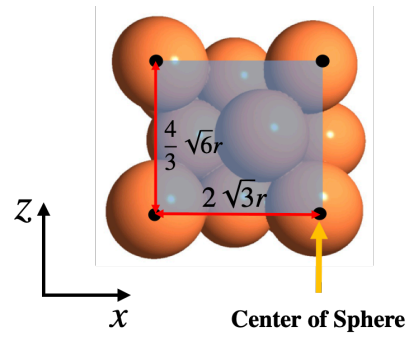

(a)

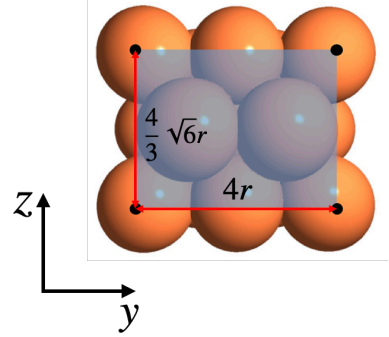

(b)



(c)

Figure 13: (a) Front, (b) side and (c) plan views of Fig. 10. The colored rectangular part is extracted for generating the unit model shown in Fig. 12.

\section{Voxel Based Solid Modelling}

The open cell model described in the previous section is midair one, i.e., the inside of the model is empty. In this section, to extend application fields of the open cell model, we show a method for converting the midair model to solid one. In particular, we consider constructing the solid model as a voxel mesh. This is because, by employing the voxel mesh, the solid model is generated as simple and convenient one. For example, the model can be applied to FEM simulations. In addition, an arbitrary shape as constructed by the open cell model can be generated.

To generate the voxel mesh, the colored rectangular domain shown in Fig. 13 is first divided into $N \times M \times L$ rectangle cells, where $N, M$ and $L$ denote the numbers of divisions for $x$-, $y$ - and $z$-direction, respectively. Note that the size of all cells is the same. The voxel mesh is generated by erasing redundant cells. To recognize the redundant cells, the implicit function $f(\boldsymbol{x})$ for generating the midair model and the property described as (1) are employed. Namely, the value of $f(\boldsymbol{x})$ in the gravity point of each cell is calculated, and if the value is negative, the cell is erased. Figure 16(a) shows the voxel mesh converted from the unit model shown in Fig. 12. Note that the periodic structure retains in the voxel mesh 


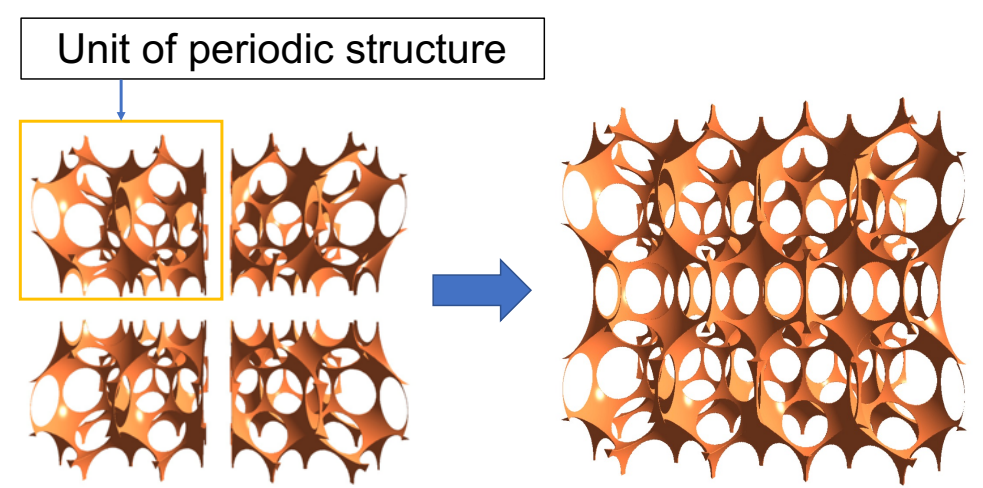

Figure 14: Schematic view of an extended open cell model by connecting the unit model shown in Fig. 12.

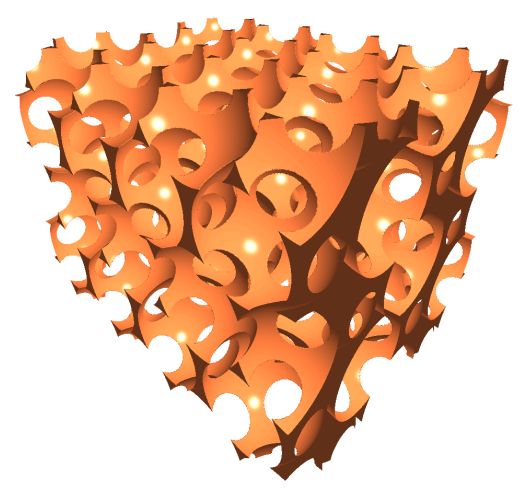

Figure 15: An open cell model extended for $x$-, $y$ - and $z$-direction by the unit model.

shown in Fig. 16(a). Namely, Fig. 16(a) is a unit voxel mesh of the open cell model. Hence, this can be connected similarly with Fig. 14. Naturally, an open cell model represented by voxel mesh can arbitrarily be extended as shown in Fig. 16(b).

Finally, we describe a strategy for generating an arbitrary shape constructed by voxel mesh of the open cell model. We assume that the shape is defined as an implicit surface $g(\boldsymbol{x})=0$. In addition, we also assume that the implicit surface is contained by the voxel mesh extended by the unit model shown in Fig. 16. The schematic view of these assumptions are shown in the left side of Fig. 17. The implicit surface is converted to a voxel mesh of the open cell model, based on the value of the implicit function $g(\boldsymbol{x})$. Namely, the value of $g(\boldsymbol{x})$ in the gravity point of each cell in the voxel mesh is calculated, and the shape is constructed as a collection of the cells in which the value is positive or zero. The schematic view for extracted cells of voxel mesh is shown in the right side of Fig. 17. Here, we demonstrate three results for complex shaped model generated by the strategy. Figures 18(a), (b) and (c) show implicit surfaces generated from the point data for Bunny, Dragon and Lucy models [9], respectively. The shapes constructed by voxel mesh of the open cell model for Figs. 18(a), (b) and (c) are shown in Figs. 19(a), (b) and (c), respectively. We see from Figs. 19(a), (b) and (c) that the 


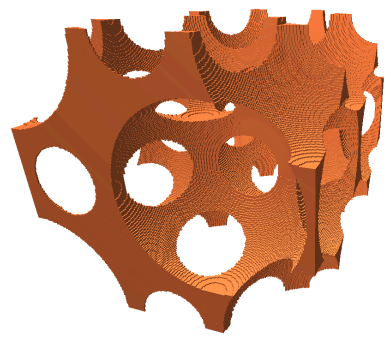

(a)

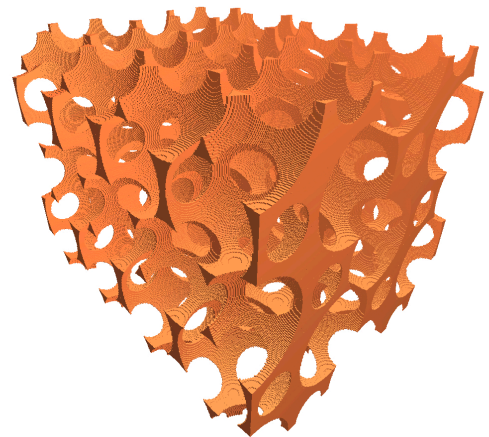

(b)

Figure 16: Voxel meshes converted from (a) the unit model shown in Fig. 12 and (b) the extended model shown in Fig. 15.
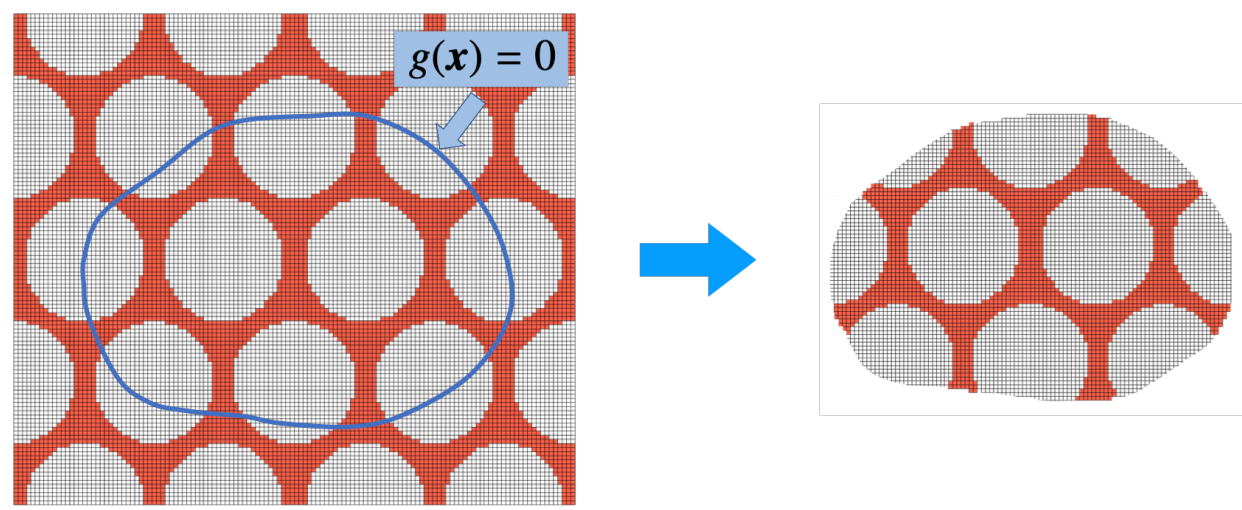

Figure 17: Schematic view of generating an arbitrary shape constructed by voxel mesh of the open cell model, assuming the shape is defined as an implicit surface $g(\boldsymbol{x})=0$.

shapes are extracted based on the implicit surfaces represented in Figs. 18(a), (b) and (c), respectively. Note that some unconnected cells sometimes occur, e.g., parts of left arm is not connected in Fig. 19(c). However, these cells can be connected by setting a smaller value of $r$.

\section{Conclusion}

To generate an open cell metal foam model simply, a sphere-function-based method has been proposed. In the proposed method, an implicit function $f(\boldsymbol{x})$ is generated by CSG of spheres that are arranged appropriately so that the Plateau borders can be constructed, and the open cell model is represented as an implicit surface, $f(\boldsymbol{x})=0$. By extracting a part of the implicit surface, a unit model of the open cell model that has a periodic structure is generated. Conclusions obtained in the present study are summarized as follows: 


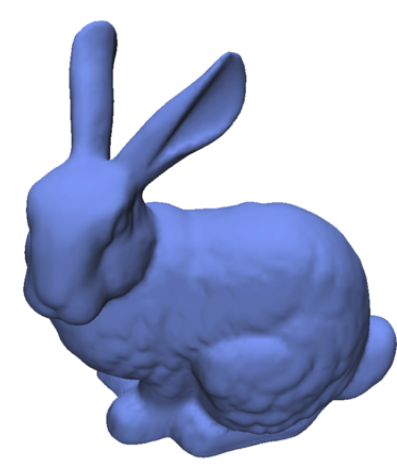

(a)

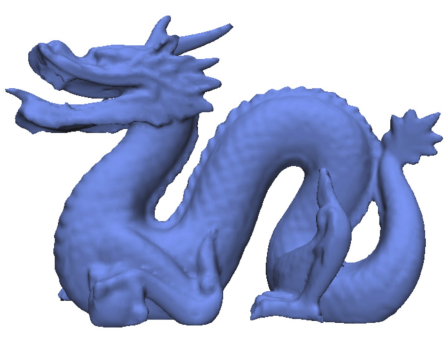

(b)

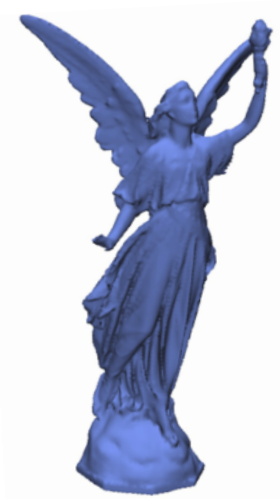

(c)

Figure 18: Implicit surfaces $g(\boldsymbol{x})=0$ for (a) Bunny, (b) Dragon and (c) Lucy models.

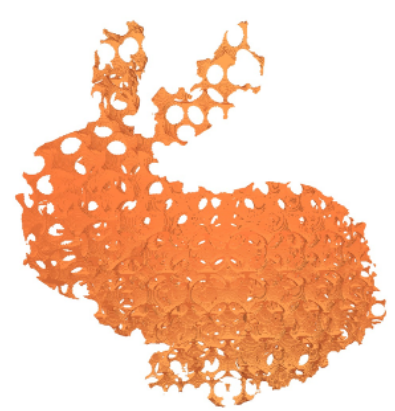

(a)

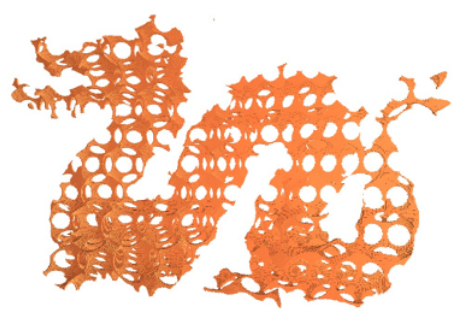

(b)

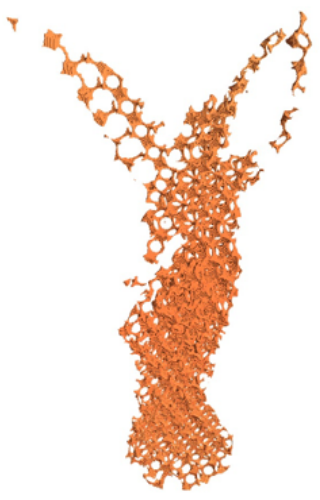

(c)

Figure 19: Extracted voxel meshes of open cell model for (a) Bunny, (b) Dragon and (c) Lucy models.

- In the open cell model generated by the proposed method, cross-sections of edges are concave triangles that are observed in those of edges in the real metal foam. Hence, the Plateau borders can be represented by the proposed method.

- The proposed method can be generalized by employing the $p$-norm-based implicit functions instead of sphere-based ones, and various sizes of Plateau borders and junctions can be constructed in the model.

- The open cell model can arbitrarily be extended by combining the unit model.

- Based on the values of $f(\boldsymbol{x})$, a voxel mesh of open cell model can be constructed with retaining the periodic structure.

- An arbitrary shape defined by an implicit surface $g(\boldsymbol{x})=0$ can be represented as a 
collection of extracted cells in the voxel mesh of open cell model.

By employing the voxel meshes of the open cell models generated by the proposed method, we have shown that arbitrary shapes can be represented. Hence, we consider that they can be applied to many kinds of simulations in which complex shaped domains exist. For evaluating the voxel meshes of open cell models, we consider applying them to simulations based on the finite element method as future work.

\section{Acknowledgement}

This work was partially supported by JSPS KAKENHI under Grant 18K11329.

\section{References}

[1] M. Ashby, T. Evans, N. A. Fleck, J. W. Hutchinson, H. N. G. Wadley, L. J. Gibson: Metal Foams: A Design Guide, Butterworth-Heinemann, Oxford, 2000.

[2] F. García-Moreno: Commercial applications of metal foams: Their properties and production, MDPI, 9:2 (2016), Art. no. 85.

[3] J. Bloomenthal, C. Bajaj, J. Blinn, M. P. Cani-Gascuel, A. Rockwood, B. Wyvill ,G. Wyvill: Introduction to Implicit Surfaces, Morgan Kaufmann, San Francisco, 1997.

[4] Y. Hanaoka, M. Nojiri, T. Itoh, S. Nakata, K. Watanabe: Three-dimensional shape modelling of metal foam with rounded cells by implicit surfaces, JASSE, 6:1 (2019), 195-214.

[5] B. Sonon, B. Francois, T. J. Massart: An advanced approach for the generation of complex cellular material representative volume elements using distance fields and level sets, Computational Mechanics, 56:2 (2015), 221-242.

[6] D. Weaire, S. Hutzler: The Physics of Foams, Oxford University Press Inc., New York, 1999.

[7] N. J. Mills: The high strain mechanical response of the wet Kelvin model for open-cell foams, International Journal of Solids and Structures, 44:1 (2007), 51-65.

[8] J. C. Hart: Sphere tracing: A geometric method for the antialiased ray tracing of implicit surfaces, The Visual Computer, 12:10 (1996), 527-545.

[9] The Stanford 3D Scanning Repository, accessed on Apr. 7, 2020. [Online]. Available: http://graphics.stanford.edu/data/3Dscanrep/ 